\title{
Mechanisms of resistance to a PI3K inhibitor in gastrointestinal stromal tumors: an omic approach to identify novel druggable targets
}

This article was published in the following Dove Press journal: Cancer Management and Research

\author{
Gloria Ravegnini ${ }^{1} * *$ \\ Giulia Sammarini ${ }^{1} *$ \\ Sebastian Moran ${ }^{2}$ \\ Giovanni Calice ${ }^{3}$ \\ Valentina Indio ${ }^{4}$ \\ Milena Urbini ${ }^{4}$ \\ Annalisa Astolfi ${ }^{4}$ \\ Federica Zanotti ${ }^{1}$ \\ Maria A Pantaleo ${ }^{4,5}$ \\ Patrizia Hrelia' \\ Sabrina Angelini ${ }^{1}$ \\ 'Department of Pharmacy and \\ Biotechnology, University of Bologna, \\ Bologna, Italy; ${ }^{2}$ Cancer Epigenetics and \\ Biology Program (PEBC), Bellvitge \\ Biomedical Research Institue (Idibell), \\ I'Hospitalet de Llobregat, Barcelona, \\ Spain; ${ }^{3}$ Laboratory of Preclinical and \\ Translational Research, IRCCS-CROB, \\ Referral Cancer Center of Basilicata, \\ Rionero in Vulture, Italy; ${ }^{4}$ Giorgio Prodi \\ Cancer Research Center, University of \\ Bologna, Bologna, Italy; ${ }^{5}$ Department of \\ Specialized, Experimental, and Diagnostic \\ Medicine, Sant'Orsola-Malpighi Hospital, \\ University of Bologna, Bologna, Italy
}

*These authors contributed equally to this work

Correspondence: Sabrina Angelini Department of Pharmacy and Biotechnology, University of Bologna, 48 Via Irnerio, Bologna 40126, Italy

Tel +3905I $209 \quad 1787$

Fax +39 05। 209 I780

Email s.angelini@unibo.it
Background: Gastrointestinal stromal tumors (GISTs) represent a worldwide paradigm of target therapy. The introduction of tyrosine kinase inhibitors has deeply changed the prognosis of GIST patients, however, the majority of them acquire secondary mutations and progress. Unfortunately, besides tyrosine-kinase inhibitors, no other therapeutic options are available. Therefore, it is mandatory to identify novel molecules and/or strategies to overcome the inevitable resistance. In this context, after promising preclinical data on the novel PI3K inhibitor BYL719, the NCT01735968 trial in GIST patients who had previously failed treatment with imatinib and sunitinib started. BYL719 has attracted our attention, and we comprehensively characterized genomic and transcriptomic changes taking place during resistance.

Methods: For this purpose, we generated two in vitro GIST models of acquired resistance to BYL719 and performed an omic-based analysis by integrating RNA-sequencing, miRNA, and methylation profiles in sensitive and resistant cells.

Results: We identified novel epigenomic mechanisms of pharmacological resistance in GISTs suggesting the existence of pathways involved in drug resistance and alternatively acquired mutations. Therefore, epigenomics should be taken into account as an alternative adaptive mechanism.

Conclusion: Despite the fact that currently we do not have patients in treatment with BYL719 to verify this hypothesis, the most intriguing result is the involvement of H19 and PSTA1 in GIST resistance, which might represent druggable targets.

Keywords: gastrointestinal stromal tumors, GIST, BYL719, PI3K inhibitor, tyrosine-kinase inhibitors

\section{Introduction}

Gastrointestinal stromal tumors (GISTs) are rare mesenchymal tumors harboring KIT/PDGFRA-driver mutations in $85 \%-90 \%$ of cases. ${ }^{1}$ GISTs are the example par excellence of targeted therapy in solid tumors. Indeed, with the introduction of the tyrosine-kinase inhibitor (TKI) imatinib, patients' prognosis have dramatically improved. ${ }^{2}$ Despite imatinib effectiveness, the majority of patients with advanced GISTs have persistent measurable disease and eventually develop progressive disease within 24-36 months. ${ }^{3}$ About 20 years after its approval, imatinib is still the gold standard in GIST treatment. However, to cope with the emergence of pharmacological resistance, the multi-TKIs sunitinib and regorafenib have been introduced, as second- and third-line treatment, respectively, in GIST management. Sunitinib and regorafenib share a mechanism of action with imatinib, adding 
clinical benefit to GIST patients after imatinib failure. Even with clinical aids, the majority of patients experience tumor progression, due to the emergence of multiple drugresistant KIT/PDGFRA mutations. ${ }^{4-9}$ Unfortunately, all the approved treatments for GIST management are TKIs, and to date there are no additional therapeutic options. Therefore, the identification of novel druggable targets, favored by better characterization of the resistance process, may represent a key starting point to achieve a different clinical approach. In this regard, a novel PI3K inhibitor — BYL719 (Novartis) — is currently being tested in a phase IB trial in GIST patients who have previously failed imatinib and sunitinib (NCT01735968). ${ }^{10}$ Specifically, BYL719 is a selective inhibitor of the PI3K catalytic p110a subunit. ${ }^{11}$ Indeed, the PI3K pathway, which is downstream of KIT/ PDGFRA receptors, is frequently activated in GIST and thought to be related to imatinib resistance. ${ }^{12-16}$ Therefore, PI3K-pathway inhibition represents an attractive target and a promising strategy to counteract imatinib resistance in GIST. In this study, we comprehensively characterized genomic and transcriptomic changes taking place during resistance and how GIST cells evolve from being drugsensitive to drug-resistant, from an "omic" point of view. For this purpose, we generated two in vitro GIST models of acquired resistance to BYL719 and performed an omicbased analysis by integrating RNA sequencing, miRNA profiling, and methylation profiling in sensitive and resistant cells.

\section{Methods}

\section{Cell culture and treatment}

Two established human imatinib-resistant GIST cell lines, GIST48 and GIST48B, were used (Table 1). These were authenticated by KIT-sequencing and TKI-sensitivity experiments, routinely grown in adhesion, and cultured at $37^{\circ} \mathrm{C}$ in a $5 \% \mathrm{CO}_{2}$ humidified atmosphere. To generate BYL719-resistant sublines, GIST48 and GIST48B were exposed to increasing concentrations of BYL719 (Selleck Chemicals, Houston, TX, USA), starting with a concentration of $0.05 \mu \mathrm{M}$ and increasing gradually to 5 $\mu \mathrm{M}$. Fresh drug was provided every 3-4 days, when the medium was replaced. After the cells had acquired the ability to grow in the presence of a specific concentration of BYL719, a proportion of them was frozen and the remaining grown at the next-highest drug level. After approximately 50 weeks, sublines of cells growing in 5 $\mu \mathrm{M}$ BYL719 were maintained continuously in culture at this dose. Upon receipt and before each change of BYL719 concentration, GIST lines were tested for mycoplasma contamination and were found to be negative.

\section{$\mathrm{IC}_{50}$ : MTT-assay method}

$\mathrm{IC}_{50}$ was evaluated by MTT assays using a standard protocol. ${ }^{17}$ Briefly, $10^{4}$ cells/well were seeded in triplicate in a 96-well plate and incubated for 24 hours. After incubation, the medium was removed and replaced with a fresh one containing BYL719 in serial dilution and incubated for 48 hours. Subsequently, cells were washed with PBS and incubated with MTT $(5 \mathrm{mg} / \mathrm{mL})$ in PBS for 2 hours. Following MTT removal, the formazan crystals were dissolved in isopropanol and absorbance measured at $570 \mathrm{~nm}$ with a Tecan spectrophotometer (Spectra Model Classic, Salzburg, Austria).

\section{DNA and RNA isolation}

Genomic DNA and total RNA were extracted from parental (GIST48 and GIST48B) and BYL719-resistant (GIST48-R and GIST48B-R) cultured cells using the QIAamp DNA minikit and RNeasy minikit, respectively,

Table I GIST cell-lines ${ }^{\text {a }}$ characteristics

\begin{tabular}{|l|l|l|l|}
\hline & Origin & KIT mutations and other characteristics & $\begin{array}{l}\text { Growth } \\
\text { medium }^{\mathbf{b}}\end{array}$ \\
\hline GIST48 & $\begin{array}{l}\text { GIST } \\
\text { primary/ }^{\text {patient }}{ }^{\mathrm{C}} \\
\text { GIST48B } \\
\text { Subline of } \\
\text { GIST48 }\end{array}$ & $\begin{array}{l}\text { Primary, homozygous KIT exon II (V560D) mutation; secondary, heterozygous KIT exon I7 } \\
\left(\mathrm{D} 820 \mathrm{~A}^{\mathrm{d}}\right) \text { mutation. } \\
\text { Retains primary mutation (KIT V560D) in all cells; } \\
\text { nearly undetectable KIT transcript and protein; secondary, heterozygous KIT exon I7 (D820A } \\
\text { mutation; keeps downstream KIT signaling active }\end{array}$ & $\begin{array}{l}\text { IMDM + I5\% } \\
\text { FBS } \\
\text { FBS }\end{array}$ \\
\hline
\end{tabular}

Notes: ${ }^{a}$ Kindly provided by Dr Fletcher (Department of Pathology, Brigham and Women's Hospital, Harvard Medical School, Boston, MA); ${ }^{\mathrm{b}}$ reagents purchased from Thermo Fisher Scientific (Waltham, MA); ${ }^{c}$ established from a GIST patient that had progressed after initial response to imatinib therapy; ${ }^{\mathrm{d}}$ located in the kinase-activation loop, confers resistance to imatinib and sunitinib.

Abbreviations: IMDM, Iscove's Modified Dulbecco's Medium; FBS, Fetal Bovine Serum. 
following manufacturer's instructions. Both kits were purchased from Qiagen (Hilden, Germany).

\section{KIT and PI3KCA mutational analysis}

Genomic DNA from parental and BYL719-resistant cell lines was screened for the presence of hot-spot mutations in KIT (exons 9, 11 13, 14, 17, and 18) and PI3KCA (exons 9 and 20). Selected exons were amplified by PCR using specific primers. Amplified PCR products were purified and sequenced on both strands using a Big Dye Terminator version 1.1 cycle-sequencing kit (Thermo Fisher Scientific). Sanger sequencing was performed on an ABI 310 genetic analyzer (Applied Biosystems) and analyzed using Chromas (Technelysium). Sequencing data were analyzed using BLAST (https://blast.ncbi.nlm.nih. gov/Blast.cgi) to match sequences with reference sequences of KIT (NM_000222) and PI3KCA (NM_006218) genes obtained from Ensembl genome browser 94 (https://www.ensembl.org/index.html).

\section{Western blot analysis}

Whole-cell protein lysates were prepared from parental and BYL719-resistant cell-monolayers using NP40 buffer containing protease inhibitors (Halt protease- and phosphatase-inhibitor cocktail; Thermo Fisher Scientific) and $1 \mathrm{mM}$ phenylmethylsulfonyl fluoride (Sigma-Aldrich). Proteins were separated on SDS-PAGE and transferred onto nitrocellulose membranes. Transfer efficiency was demonstrated by Ponceau S staining (Sigma-Aldrich). Membranes were blocked by $5 \%$ skimmed milk, followed by incubation at $4^{\circ} \mathrm{C}$ overnight with the presence of a primary antibody against KIT (A4502; Dako, Ely, UK), phospo-KIT (3391; Cell Signaling Technology, Leiden, Netherlands), AKT (9272; Cell Signaling Technology), phospo-AKT (9271; Cell Signaling Technology), MAPK (9102; Cell Signaling Technology), phospo-MAPK (9101; Cell Signaling Technology), mTOR (2972; Cell Signaling Technology), phospo-mTOR (2448; Cell Signaling Technology), PTEN (138G6; Cell Signaling Technology), and actin (A1978; Sigma-Aldrich). After rinsing, membranes were incubated with horseradish peroxidase-conjugated secondary antibody (Thermo Fisher Scientific) at room temperature for 2 hours. After further rinsing, immunoreactive bands were visualized by enhanced chemiluminescence (BioRad, Hercules, CA, USA) and signals captured and quantified using ChemiDoc (BioRad).

\section{$A B C$ transporter gene-expression analysis}

Total RNA was reverse-transcribed to cDNA using a high capacity RNA-to-cDNA kit (Applied Biosystems) according to the manufacturer's instructions. cDNA was loaded into a TaqMan human ABC-transporter array, which allows quantitative gene-expression analysis of human ABC-transporter genes important in drug discovery and resistance. In particular, it contains assays in triplicate for 50 human genes and 14 endogenous controls in a 348-well array. mRNA-expression levels were normalized using GAPDH and $18 \mathrm{~S}$ as endogenous controls, and data were analyzed using the $2^{-\Delta \Delta \mathrm{Ct}}$ method. mRNAs with $\mathrm{Ct}>35$ were considered unexpressed and excluded from further analysis.

\section{RNA sequencing (RNA-seq)}

Whole-transcriptome RNA libraries were prepared in accordance with the TruSeq RNA Sample Prep version 2 protocol (Illumina, San Diego, CA, USA). Poly(A)RNA molecules from 500 ng total RNA were purified using oligo-dT magnetic beads. Subsequently, mRNA was fragmented and randomly primed for reverse transcription followed by second-strand synthesis to generate double-stranded cDNA fragments. The cDNA fragments generated went through a terminal end-repair process and ligation using paired-end sequencing adapters. The obtained products were amplified to enrich for fragments carrying adapters ligated on both ends, and to add additional sequences complementary to the oligonucleotides on the flow cell, thus creating the final cDNA library. $12 \mathrm{pM}$ paired-end libraries were amplified and ligated to the flow cell by bridge $\mathrm{PC}$ and sequenced at $2 \times 75 \mathrm{bp}$ read length for RNA using Illumina sequencing-by-synthesis technology.

\section{RNA-seq : bioinformatic analysis}

After demultiplexing and FASTQ generation, through the bcltofastq function developed by Illumina, paired-end reads were trimmed using AdapterRemoval (https:// github.com/MikkelSchubert/adapterremoval) with the aim of removing stretches of low-quality bases $(<Q 10)$ and Truseq/Nextera rapid-capture adapters present in the sequences. Sequences coming from RNA-seqwere mapped with TopHat/BowTie pipeline and PCR or optical duplicates were removed with the function rmdup of SAMtools. 


\section{RNA-seq: SNV calling}

Single nucleotide variant (SNV) calling was performed with SAMtools and SNVMix2, which allows the identification of all point mutations and insertion/deletion variants. Variants in the dbSNP, 1000 Genomes, ExAc, and EVS databases with frequency $>1 \%$ were excluded. The possible functional effects of identified variants were analyzed with three in silico tools: SIFT, PolyPhen2, and MutationTaster2. ${ }^{18-20}$

\section{Gene-expression analysis}

In order to compare gene-expression profile (GEP) between BYL719-sensitive and -resistant GIST cell lines, RNA-seq data were analyzed. After the alignment procedure, the BAM file obtained was manipulated with SAMtools to remove the optical/PCR duplicate and to sort and index it. The HTSeq count (Python HTSeq package) was adopted to count the number of reads mapped on known genes included in Ensembl 72 annotation features.

\section{Functional annotation, GO, and pathway analysis}

NetworkAnalyst (https://www.networkanalyst.ca/faces/ home.xhtml) was used to identify molecular pathways and functional groupings. Gene-interaction networks, biofunction, and pathway analysis were generated using differentially expressed genes (DEGs) into known functions, pathways, and networks, primarily based on human studies. The DEGs were organized in Gene Ontology (GO) biofunction and regulatory effect networks. Significance was set at $P<0.05$. NetworkAnalyst uses a comprehensive high-quality protein-protein interaction database based on InnateDB. This database contains manually curated protein-interaction data from published literature, as well as experimental data from several protein-protein interaction databases, including IntAct, MINT, DIP, BIND, and BioGRID. The database currently contains 14,755 proteins and 145,955 interactions for humans.

\section{qRT-PCR}

Gene-expression levels of H19 and PSAT1 were evaluated through quantitative reverse-transcription (qRT) PCR. Briefly, RNA from both parental and BYL719resistant cell lines was reverse-transcribed to cDNA using a high-capacity RNA-to-cDNA kit (Applied Biosystems) according to the manufacturer's instructions. qRT-PCR was performed with a Fast SYBR Green
Master Mix (Applied Biosystems) using the 7900HT realtime PCR system. qRT-PCR assays for H19 and PSAT1 were performed using the primers H19-Fwd 5'ATCGGTGCCTCAGCGTTCGG-3', H19-Rev 5'-CTGTC CTCGCCGTCACACCG-3', PSAT1-Fw 5'-ATACAGA GAATCTTGTGCGGG-3', PSAT1-Rev 5'-CATAGTCAG CACACCTTCCTG-3', GAPDH-Fwd 5'CGGGAAGCTT GTCATCAAT-3', and GAPDH-Rev 5'-GACTCCACGAC GTACTCAGC-3'. All primers were obtained from Integrated DNA Technologies. Relative expression levels were evaluated by the $2^{-\Delta \Delta \mathrm{Ct}}$ method using $G A P D H$ as a housekeeping gene.

\section{miRNA-expression evaluation}

Total RNA (300 ng) was reverse-transcribed using a TaqMan miRNA reverse-transcription kit (Thermo Fisher Scientific) using Megaplex RT primers Human Pool A and Pool B. cDNAs were load on the TaqMan arrays Human MicroRNA A and B Cards and run on a 7900HT real-time PCR system in accordance with the manufacturer's procedure. miRNA data were analyzed with SDS relative quantification software version 2.4 (Applied Biosystems) and miRNAs with $\mathrm{Ct}>35$ were considered unexpressed and excluded from further analysis. miRNA-expression levels were normalized using U6 and RNU48 as endogenous controls. Normalization was carried out by subtracting the mean $\mathrm{Ct}$ from individual $\mathrm{Ct}$ values. R-Bioconductor (package Limma) was adopted to evaluate differential expression profiles between the parental and BYL719-resistant GIST cell lines.

\section{Global methylation profile}

Genomic DNA (600 ng/sample) were bisulfite-converted using EZ DNA methylation kits (Zymo Research), and DNA methylation was measured using the Illumina Infinium HD-methylation assay with Infinium Methylation Epic BeadChips according to Illumina's protocol. Raw data (Idat files) were processed in R Bioconductor (minfi27). The quality of each sample was analyzed and probe signals removed when: i) $P>0.05$; ii) $>1 \%$ of the data set contained no data; or iii) probes contained single-nucleotide polymorphisms. None of the samples included in the study was flagged as an outlier. ${ }^{21}$ Statistical analyses were carried out using GenomeStudio, normalizing Idat values with controls provided by Illumina. The methylation score of each $\mathrm{CpG}$ is represented as a $\beta$-value, and differences between $\beta$-values of treated and untreated cells represent alterations in methylation level. The CpGs selected were those with absolute 
methylation differential value of $>0.2$ or $<-0.2 .^{21,22}$ To identify CpGs on promoter regions, we considered only UCSC reference-gene groups TSS200 or TSS1500.

\section{Identification of validated miRNA targets}

Targets of significant miRNAs were identified through specific in silico tools that allowed prediction of the most probable targets. Specifically, to limit false-positive results, we used the miRTarBase tool, which encloses more than 400,000 miRNA-target experimentally verified interactions, collected by manually surveying pertinent literature after systematic data mining of the text.

\section{miRNA-profile and gene-expression correlation}

miRNA and mRNA arrays were analyzed to highlight pairs of mRNAs and miRNAs that were discordant (up- versus downregulated and vice versa). Potential miRNA-mRNA interactions and miRNA/mRNA-expression profiles were used to construct functional interaction networks.

\section{Methylation-profile and gene-expression correlation}

To integrate methylation profiles and GEP, we considered only $\mathrm{CpGs}$ on promoter regions that shown absolute methylation differentials of $>0.2$ or $<-0.2$ in parental lines versus BYL719-resistant ones. Promoter regions were defined as upstream 1,500 bp and downstream 200 bp from the transcription-start site (TSS) of each gene. For each differentially methylated gene, we checked the expression level derived from RNA sequencing.

\section{Results}

\section{MTT assay}

GIST48 and GIST48B cell lines were exposed to BYL719 using doses that were increased in a stepwise manner. We thus established two BYL719-resistant GIST cell lines (48-R and 48B-R), which exhibited BYL719 IC $_{50}$ values that were about 15-fold higher than the parental cell lines (Figure S1).

\section{KIT and PI3KCA mutational analysis}

The involvement of KIT and PI3KCA mutations as mechanisms driving BYL719 resistance in GIST cells in vitro was investigated. Mutational analysis did not reveal additional KIT mutations in BYL719-resistant cell lines by Sanger sequencing or SNV calling. With regard to PI3KCA, we Sanger sequenced exons 9 and 20, both codifying for the catalytic p110a subunit and associated with drug resistance. ${ }^{23-26}$ All samples were wild type. PI3KCA wildtype status was also confirmed by SNV calling.

\section{Western blot analysis}

To better characterize the BYL719-resistant GIST48-R and GIST48B-R lines, we performed Western blot analysis, evaluating in particular the signaling pathway downstream of KIT (Figure 1A). In the imatinib-resistant GIST48 line (as expected, considering that the targeted PI3K is downstream of KIT), we observed comparable KIT phosphorylation between GIST48 and GIST48B-R (Figure 1B). Absence of KIT phosphorylation was observed, as expected, in both GIST48B and GIST48BR. Indeed this GIST model had entirely lost KIT expression (ie, KIT-negative, despite activation of downstream signaling [Figure 1C]). No differences were observed in PTEN status between parental and BYL719-resistant cell lines. On the contrary, in both GIST models, we detected activated AKT in BYL719-sensitive cell lines and inactivation in the resistant ones. We also observed activation of mTOR in both parental and BYL719-resistant cell lines. Finally, we observed MAPK activation in both BYLsensitive and -resistant GIST models. All immunoblots are shown in Figure 1, B and C.

\section{RNA-seq: SNV calling}

We identified ten novel mutations in BYL719-resistant cells compared to parental cell lines. In particular, four mutations were identified in GIST48-R cells and six in GIST48B-R (Table 2). None of the mutations was common to both the two resistant GIST cell lines. With regard to GIST48-R, one mutation was identified as benign $(K I F C 2)$ and one probably damaging $(R P G R)$ by the three tools. One mutation (RIF1) was predicted as probably damaging by two tools. Lastly, no agreement was reached for one mutation ( $D P F 1)$. Concerning GIST48B$\mathrm{R}$, one mutation $(F R Y)$ was predicted as probably damaging by the three tools, two mutations ( $P D G F B$ and $S Y T 14)$ were identified as benign by two tools, and no agreement was reached for two mutations (DCHS1 and EEF2KMT). Lastly, one mutation (RARS2) was a gain of stop codon. Data are reported in Table 2. However, none of them was related to the PI3KCA pathway, had been previously identified in GIST, or associated with resistance in other tumor types; therefore, we did not consider them driver mutations for BYL719 resistance. 

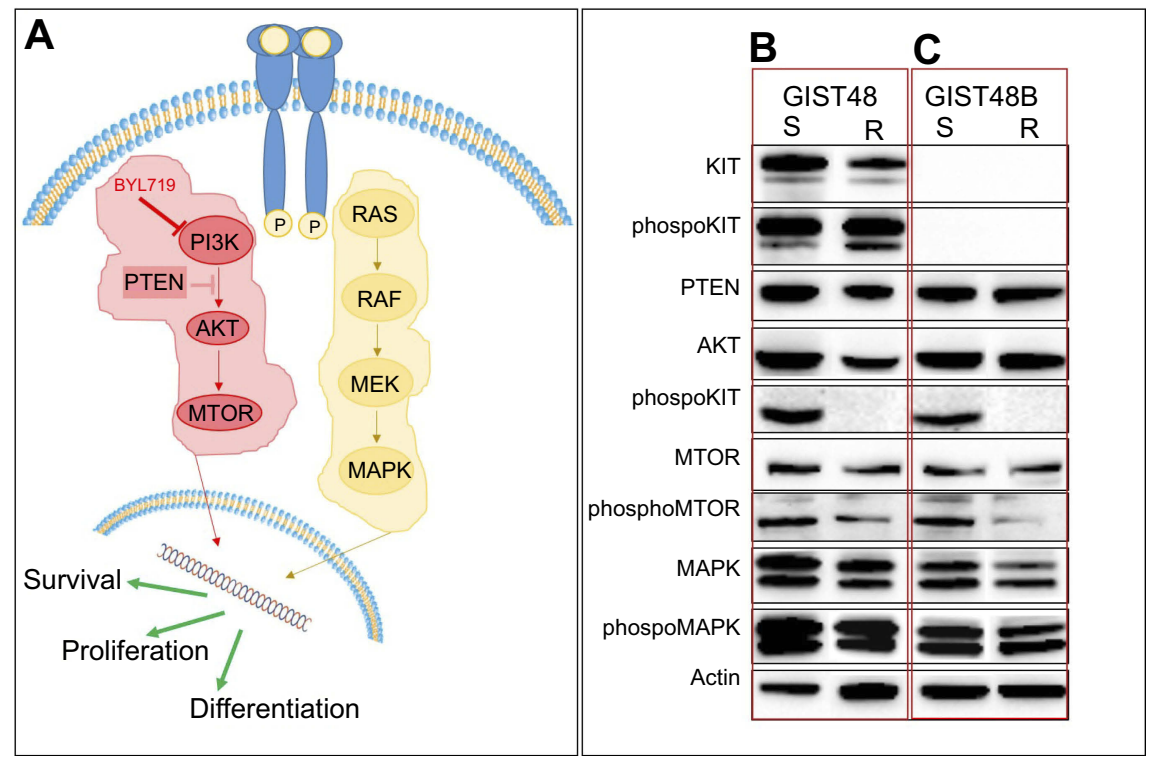

Figure I Western blot analysis.

Notes: (A) Simplified KIT-signaling pathway. Immunoblot evaluation of KIT, phospho-KIT, PTEN, AKT, phospho-AKT, mTOR, phospho-mTOR, MAPK, and phospho-MAPK in GIST48 (B) and GIST48B (C). S, BYL7I9-sensitive; R, BYL7I9-resistant.

Gene expression: $A B C$-transporter genes and RNA-seq analysis

Given the well-recognized role of ABC-transporter genes in mediating drug resistance, first we analyzed a custom panel of 50 genes in BYL719-sensitive and resistant GIST lines. None was significantly deregulated. Therefore, we performed a RNA-seq analysis, which showed 95 differentially expressed genes with $P \leq 0.001$ and a false discovery rate $<0.1$ in BYL719sensitive compared to BYL719-resistant GIST lines. In particular, 48 genes were upregulated, while 47 were downregulated. The significant DEGs are reported in Table S1. The 20 top deregulated genes -13 up- and 7 downregulated - are shown in Figure 2A. GO functional enrichment analyses of deregulated genes showed cancer, chronic myeloid leukemia, and cell cycle as the top three deregulated pathways (Kyoto Encyclopedia of Genes and Genomes pathways (KEGG), Figure 2B). In addition, we found viral reproductive process and viral reproduction to be the top biological processes involved. RNA binding, structural constituents of ribosome, and structural molecular activity were the top three molecular functions. Finally, cytosol, macromolecular complex, and ribonucleoprotein complex were the top three cellular components involved. Interestingly, among the top upregulated genes in resistant cells, there was the long non coding RNA (lncRNA) H19. RNA-seq data were confirmed by qRT-PCR, which demonstrated overexpression of H19 in GIST48-R and GIST48B-R compared with the parental BYL719-sensitive lines, with fold changes of 20.8 and 6.9 , respectively.

Table 2 Acquired mutations in BYL7I9-resistant GIST cells

\begin{tabular}{|l|l|l|l|l|l|l|l|}
\hline \multicolumn{2}{|l|}{ GIST 48 vs GIST48-R } & \multicolumn{4}{l|}{ GIST 48B vs GIST48B-R } \\
\hline Gene & Exon & AA change & Prediction* & Gene & Exon & AA change & Prediction* \\
\hline DPFI & 9 & P.C3I3G & $\mathrm{B}^{\mathrm{a},}, \mathrm{PD}^{\mathrm{c}}$ & DCHSI & 2 & p.RI68C & $\mathrm{B}^{\mathrm{a}}, \mathrm{PD}^{\mathrm{b}, \mathrm{c}}$ \\
KIFC2 & 17 & p.R732C & $\mathrm{B}^{\mathrm{a}-\mathrm{c}}$ & EEF2KMT & 2 & p.H52Q & $\mathrm{B}^{\mathrm{a}, \mathrm{b}}, \mathrm{PD}^{\mathrm{c}}$ \\
RIFI & 19 & p.V666A & PD $^{\mathrm{a}, \mathrm{c}}$ & FRY & 5 & p.DI68Y & PD $^{\mathrm{a}-\mathrm{c}}$ \\
RPGR & 6 & p.QI7IR & PD $^{\mathrm{a}-\mathrm{c}}$ & PDGFB & 3 & p.R5IH & B $^{\mathrm{a}, \mathrm{c}}$ \\
& & & & RARS2 & 1 & p.CIIX & Gain of stop codon $^{\mathrm{b}}$ \\
& & & & SYTI4 & 6 & p.V359A & $B^{\mathrm{b}, \mathrm{c}}$ \\
\hline
\end{tabular}

Note: *Prediction of the deleterious potential through ${ }^{\mathrm{a} S I F T}$, ${ }^{\mathrm{b}}$ PolyPhen, and ${ }^{\mathrm{C}}$ MutationTaster2.

Abbreviations: AA, amino acid; $B$, benign; PD, probably damaging. 


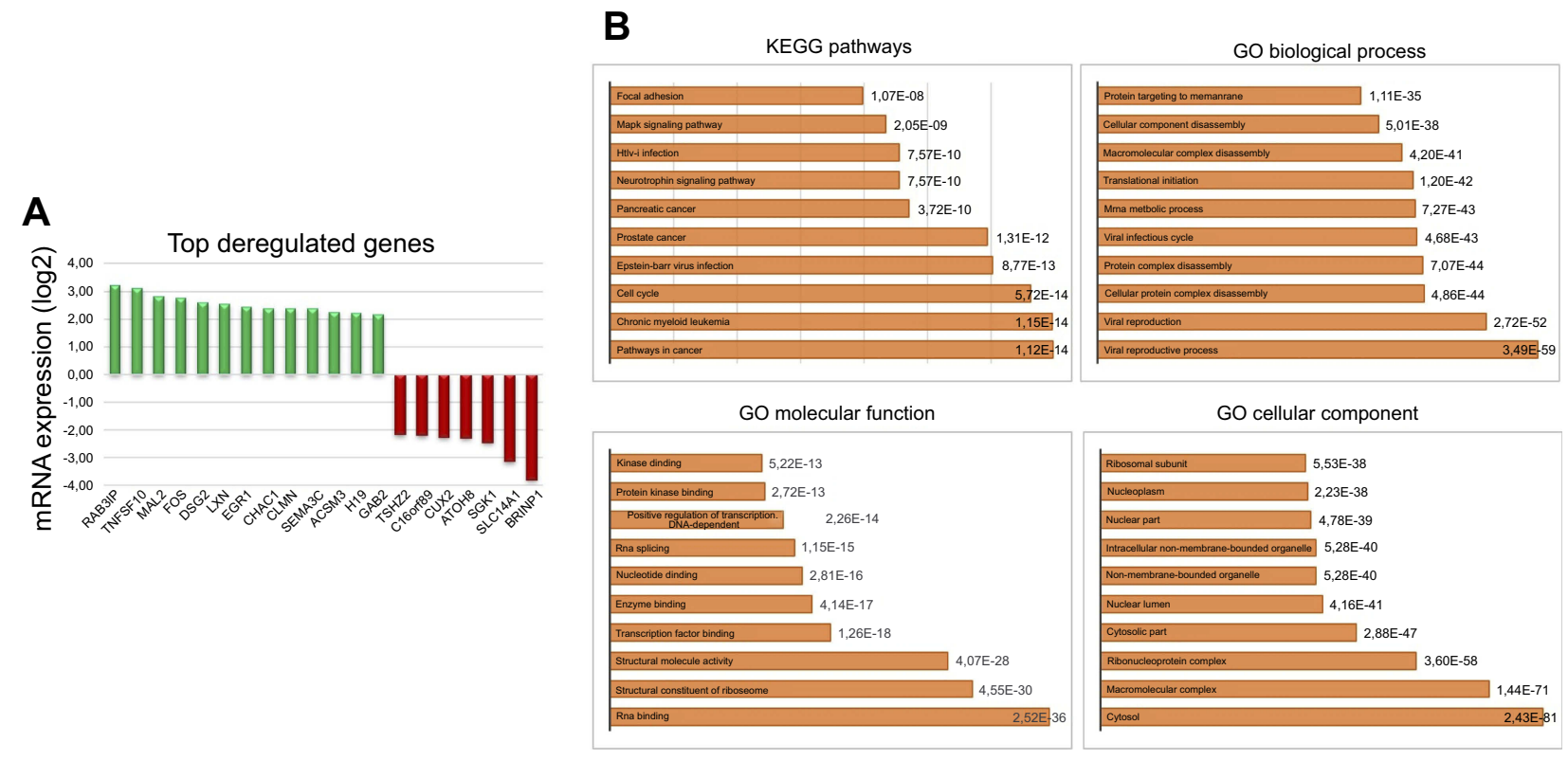

Figure 2 RNA-seq analysis.

Notes: (A) Top deregulated genes in BYL7I9-sensitive and -resistant GIST cell lines. (B) Go functional enrichment analysis of up- and downregulated genes.

Abbreviations: GIST, gastrointestinal stromal tumors; GO, gene ontology.

\section{miRNA-expression profile}

The array highlighted a total of 44 deregulated miRNAs of the 754 analyzed $(P<0.05)$; however, after adjustment, only 13 miRNAs maintained statistical significance. In particular, two miRNAs - has-miR190b and has-miR299-5p - were upregulated and eleven miRNAs downregulated in resistant GIST cell lines compared to parental ones. All differentially expressed miRNAs are reported in Table 3. Hierarchical clustering of all samples dichotomized sensitive and resistant GIST cell lines into two distinct clusters (Figure 3A). Through miRPath 3.0, we assessed miRNA regulatory roles and

Table 3 The most significantly deregulated miRNAs

\begin{tabular}{|l|c|l|}
\hline miRNA & $P$-value & $\begin{array}{l}\text { Adjusted } \\
\text { P-value }\end{array}$ \\
\hline hsa-miRI243 & $2.94^{-6}$ & 0.002232799 \\
hsa-miR520c-3p & $1.27^{-5}$ & 0.003316809 \\
hsa-miRI90b & $1.31^{-5}$ & 0.003316809 \\
hsa-miRI289 & $2.72^{-5}$ & 0.005164803 \\
hsa-miRI247-5p & 0.000118 & 0.016017348 \\
hsa-miR22-3p & 0.000126 & 0.016017348 \\
hsa-miRI267 & 0.000188 & 0.020418143 \\
hsa-miR299-5p & 0.000237 & 0.022470219 \\
hsa-miRI25b-5p & 0.000294 & 0.024789505 \\
hsa-miR656-3p & 0.000447 & 0.033993852 \\
hsa-miR33I-5p & 0.000591 & 0.040837133 \\
hsa-miRI49-3p & 0.000868 & 0.054998185 \\
hsa-miR30d-5p & 0.001126 & $0.06581247 \mid$ \\
\hline
\end{tabular}

identification of controlled pathways. ${ }^{27}$ Interestingly, among the pathways potentially modulated by the deregulated miRNAs (summarized in Figure 3B), the most significant and those with the greatest number of target genes were PI3K/AKT, MAPK, and RAS cascades, which are involved in BYL719's mechanism of action. Figure 3C shows the main miRNAs involved in cancer (highlighted in blue) and PI3KAKT (in green) and JAK-STAT (in pink) pathways. In particular, among the 13 deregulated miRNAs retrieved by our data, we identified a signature of five miRNAs mainly involved in the aforementioned pathways: miR22-3p, miR125b-5p, miR149-3p, miR190b, and miR520c-3p.

\section{Global methylation profile}

To determine whether acquired resistance involved modifications in DNA methylation, we performed genomewide DNA-methylation profiling in BYL719-sensitive and -resistant GIST cell lines. We identified 3,305 differentially methylated CpGs. Among these, 2,817 were hypermethylated and 488 hypomethylated. Of the hypermethylated CpGs, 547 were in promoter regions of 379 genes, while 102 hypomethylated sites were in promoter regions of 70 genes. Unsupervised hierarchical cluster analysis of the demethylated genes divided the sensitive and resistant cell lines into two main clusters (Figure 4). Interestingly, as shown in Figure 5, A-C, the resistant cells showed significantly more hypermethylated $\mathrm{CpG}$-island promoters compared to the sensitive counterparts. 


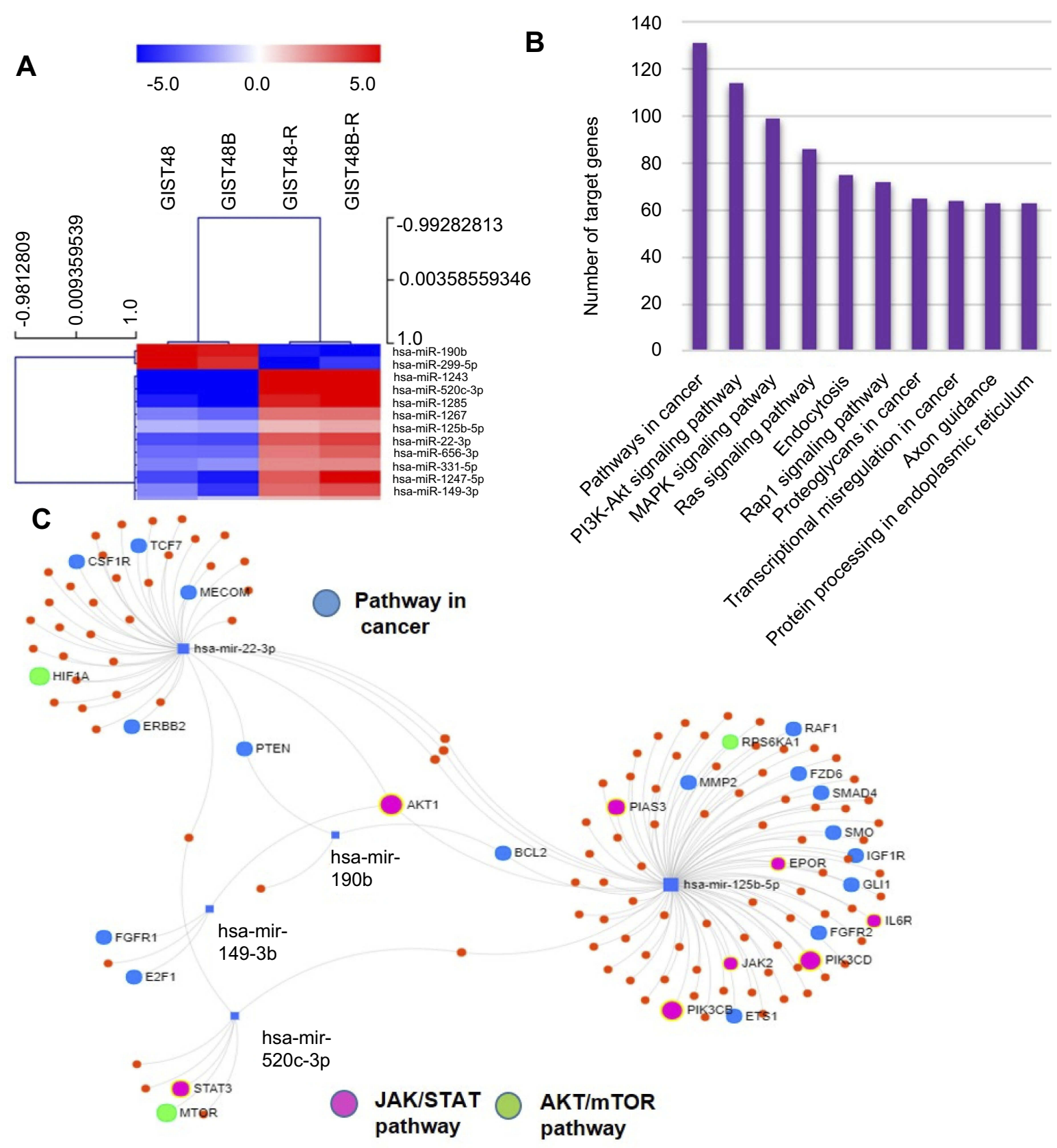

Figure 3 miRNAs profile.

Notes: (A) miRNA hierarchical clustering. miRNAs identified as differentially expressed between BYL7/9-sensitive and -resistant GIST cell lines were selected as markers for unsupervised hierarchical clustering. (B) GO functional enrichment analysis of up- and downregulated miRNAs. (C) Main miRNAs involved in cancer (blue), AKT-mTOR (green) and JAK signaling cascades (pink) pathways.

\section{miRNAs and mRNA network}

To construct miRNA-DEG networks, we downloaded the experimentally verified associations between human miRNAs and their targets from miRTarBase. This data set consists of 4,076 miRNAs and 23,054 mRNAs,${ }^{28}$ and encloses more than 400,000 miRNA-target interactions collected by manually surveying pertinent literature after systematic data mining of the text. Among the 13 deregulated miRNAs retrieved by our data, seven (miR331-5p, miR125b-5p, miR520c-3p, miR1289, miR299-5p, miR30d-5p, and miR149-3p) had verified associations with their targets. However, taking into account the canonical inverse correlation between miRNA and target expression, we were able to identify eight mRNA-miRNA networks. In particular, we found 


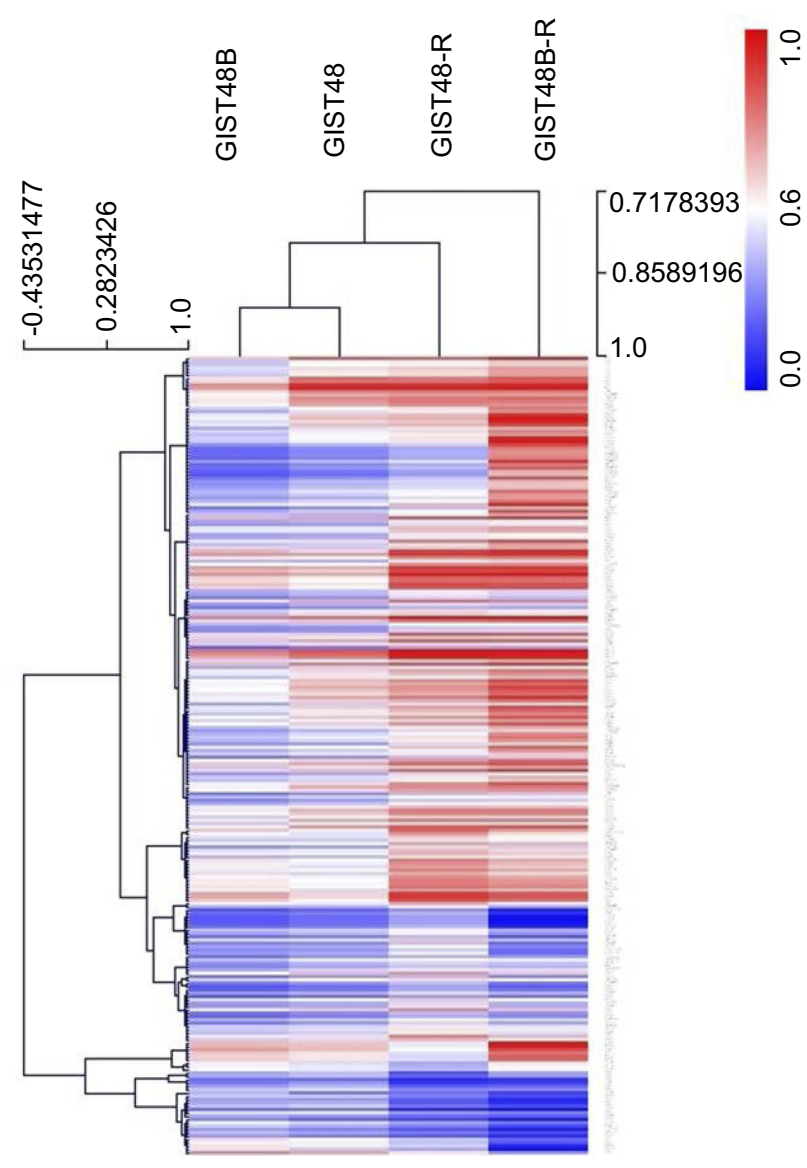

Figure 4 Global methylation profile.

Notes: Heat map showing DNA-methylation profiles in parental and BYL7I9. resistant cell lines $(\Delta \beta<-0.2$ or $>0.2)$.

four miRNAs downregulated and the corresponding seven targets overexpressed (three miRNAs targeted two distinct mRNAs). One miRNA was upregulated and the corresponding target downregulated. The miRNAs and their corresponding targets are presented in Figure 6.

\section{DNA methylation and mRNA network}

To clarify the relationship between DNA methylation and differentially expressed mRNAs, we integrated the global methylation profile with DEGs. Results are shown in Figure 7. Among the genes with the strongest promoter hypermethylation and concomitant increased expression in resistant cells were $C L M N$, a member of the hedgehoginteracting protein family MAL, which encodes the T-lymphocyte maturation-associated protein and functions in T-cell differentiation. On the contrary, promoter hypermethylation and concomitant TSHZ2 downregulation was observed, whereas PSAT1 showed strong promoter hypomethylation associated with increased gene expression after treatment. Overexpression of $P S A T 1$ was confirmed by qRT-PCR, which demonstrated overexpression of PSAT1 in GIST48-R and GIST48B-R compared with the parental BYL719-sensitive lines, with fold changes of 4.1 and 5.8, respectively.

\section{Construction of the miRNA-mRNA- DNA methylation network}

In order to identify a more comprehensive network, we integrated data deriving from GEP, miRNA, and methylation profiles. After intersection of all the data, the PSATI gene was significantly upregulated in BYL719-resistant cell lines and showed promoter hypomethylation and a potential modulation by miR125b-5p (Figure 7).

\section{Discussion}

GISTs represent a worldwide paradigm of target therapy. The introduction of imatinib to clinical practice has deeply revolutionized its management, leading GISTs from an incurable disease to a sort of chronic disease. Imatinib has determined terrific improvement in GIST prognosis; however, as often happens with TKIs, the majority of patients acquire secondary mutations and the disease progresses. Unfortunately, no therapeutic options are available for patients who have failed on imatinib and the subsequent therapeutic lines: sunitinib and regorafenib. Therefore, it is pivotal and mandatory to identify novel molecules - different from TKIs - and/or strategies to overcome the inevitable resistance. In this context, after the promising preclinical data on the novel PI3K inhibitor BYL 719, ${ }^{10}$ the NCT01735968 trial in GIST patients who had previously failed treatment with imatinib and sunitinib started. BYL719 attracted our attention, and we comprehensively characterized genomic and transcriptomic changes taking place during resistance. Understanding how GIST cells evolve from being drug-sensitive to drugresistant, from an omic point of view might accelerate the identification of novel druggable targets. For this purpose, we generated two in vitro GIST models of acquired resistance to BYL719 and performed an omic-based analysis by integrating RNA-seq, miRNA, and methylation profiling in sensitive and resistant cells. In particular, we selected GIST48 and GIST48B, sharing activation of KIT downstream signaling, including the PI3K pathway, targeted by BYL719. ${ }^{29}$ Furthermore, both cell lines are resistant to the second-line treatment sunitinib, ${ }^{30}$ making them 


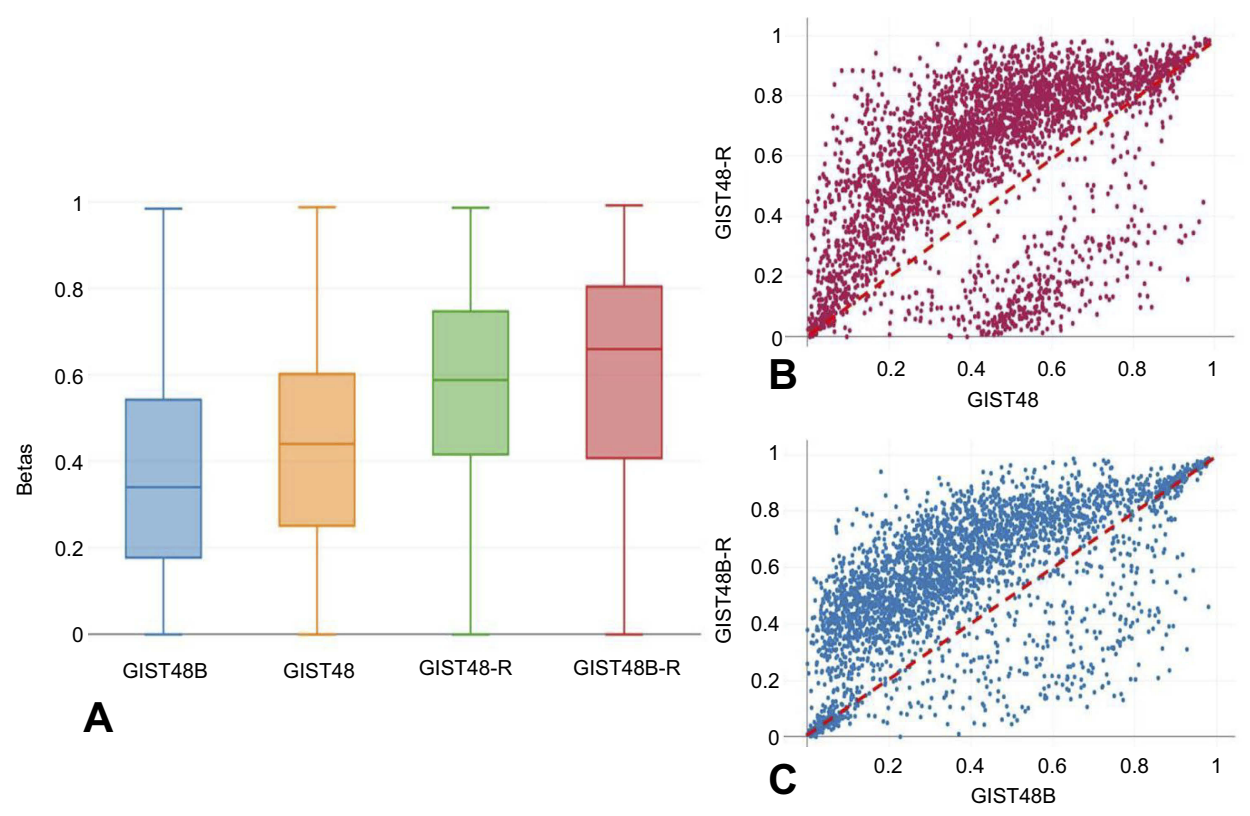

Figure 5 Global methylation profile.

Notes: (A) Box plots showing the $\beta$-values of CpGs islands at promoters only. General hypermethylation was more common in the BYL7I9-resistant cell lines. (B, C) Scatterplots of genome-wide DNA methylation levels $(\beta)$. To highlight general hypermethylation, CpG islands with $\Delta \beta<-0.2$ or $>0.2$ are shown.

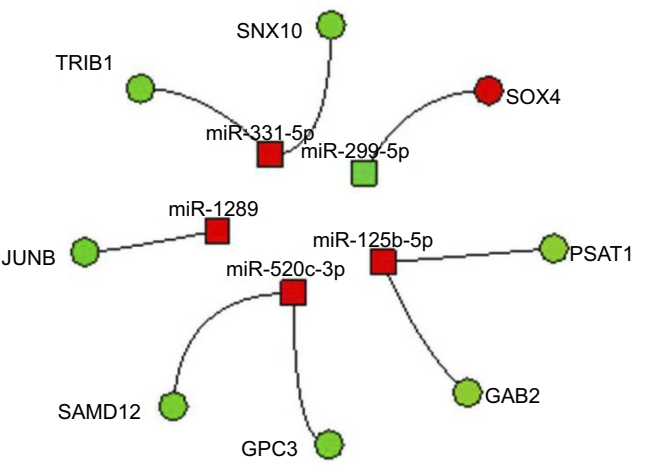

Figure 6 Integrated miRNA-mRNA regulatory networks.

Notes: Integration of GEP and miRNA profiles. Circles and squares represent genes and miRNAs, respectively. Red and green indicate downregulation and upregulation, respectively.

Abbreviation: GEP, gene expression profile.

good models, mirroring GIST patients who hypothetically will receive BYL719 treatment after imatinib and sunitinib failure.

TKI resistance is largely due to accumulation of additional kinase-domain mutations. ${ }^{31}$ Therefore, we sequenced KIT and PI3KCA hot-spot exons - in addition to RNAseq - to evaluate the appearance of novel point mutations or insertion/deletion variants possibly able to explain BYL719 resistance. Different alterations were found in BYL719-resistant lines; however, none was shared by both models, or harbored in PI3KCA and KIT genes, or downstream effectors. In addition, none of the mutated genes has been involved in drug resistance; therefore, we did not consider them driver events for BYL719 unresponsiveness, pinpointing a potential novel mechanism of resistance in GISTs. Afterward, we analyzed a precast panel of 50 well-characterized ABC-transporter genes. Overexpression of genes involved in drug efflux is among the most common mechanisms of drug resistance. ${ }^{32-34}$ However, we did not observe any significant difference in BYL719-resistant cell lines compared to sensitive ones. This prompted us to perform a more comprehensive geneexpression analysis through the RNA-seq approach. We identified the $H 19$ gene as one of the most significantly upregulated in BYL719-resistant cells compared to sensitive ones. $H 19$ has been described as an oncogenic lncRNA involved in cell proliferation, metastasis, epithelialmesenchymal transition, and poor prognosis in several cancer types, including esophageal squamous-cell carcinoma, osteosarcoma, colorectal and gastric cancer, and others. ${ }^{35-38}$

A recent study reported H19 overexpression in GIST samples compared to normal paired tissue. ${ }^{39}$ In addition, H19 upregulation showed a high correlation with ETV1 expression, which is crucial in GIST growth and survival. ETV1 cooperates with KIT through the MAPK-signaling pathway, and together they promote GIST tumorigenesis. ${ }^{40}$ Furthermore, it has been reported that H19 might promote migration and invasion in colorectal cancer by activating the RAS protein and upregulating 


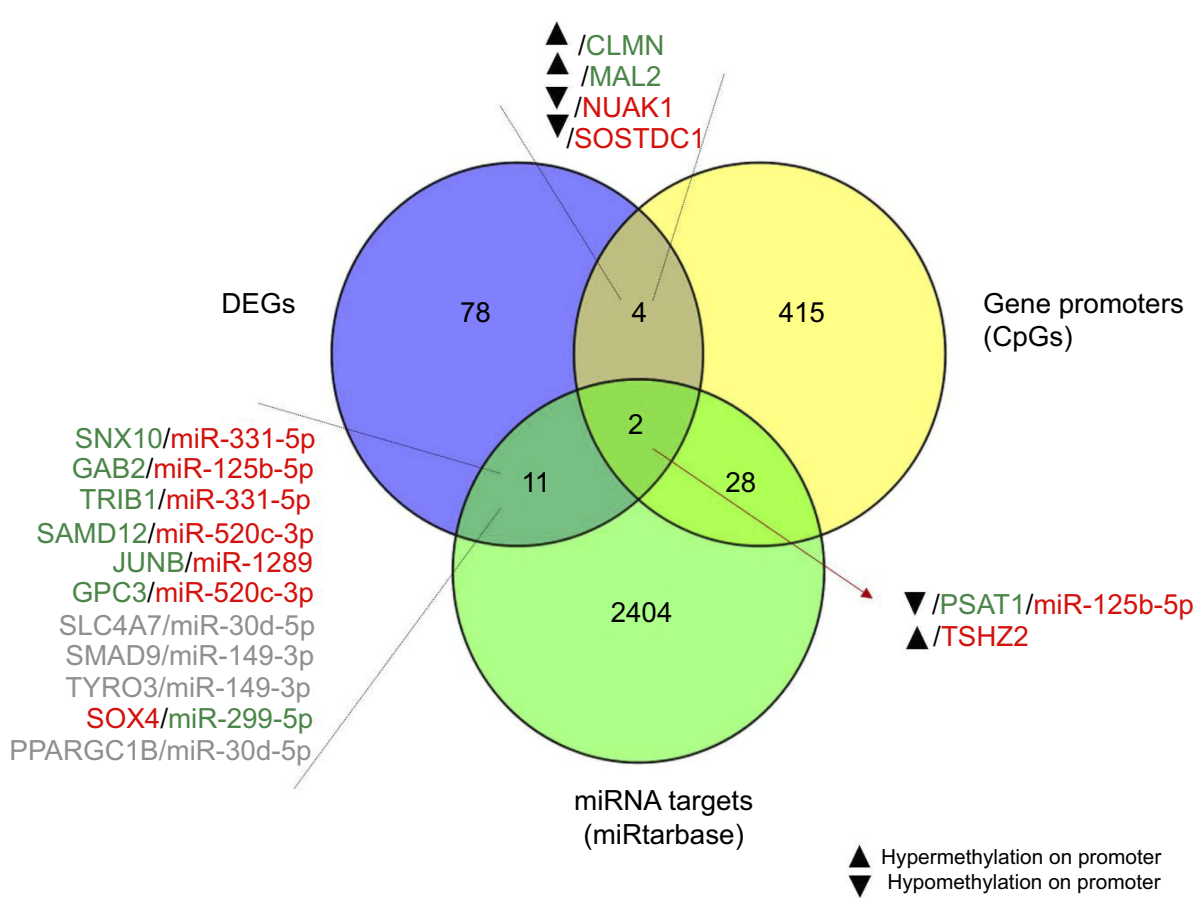

Figure 7 Integrated miRNA-mRNA-methylation regulatory networks.

Notes: Integration of GEP, miRNA, and methylation profiles. In gray are shown genes and miRNAs with the same trend of expression, which were excluded. Red and green indicate downregulation and upregulation, respectively.

Abbreviation: GEP, gene expression profile.

levels of phospho-RAF, phospho-MEK and phosphoMAPK. ${ }^{41}$ Considering that RAS is upstream of both the PI3K and MAPK pathways, ${ }^{42}$ this could represent an alternative mechanism through which resistant cells bypass PI3K and maintain their tumorigenic status. This assumption was confirmed by Western blot analysis, highlighting MAPK activation in both parental and BYL719resistant cell lines. Interestingly, we also observed a weak activation of mTOR in BYL719-resistant cell lines, despite no AKT activity. This finding agrees with the recognized complex and dynamic cross talk between PI3K/AKT and MAPK. ${ }^{43,44}$ Pathway cross talk allows a cell to achieve robust activation of key downstream targets or compensatory signaling. Particularly, the latter might be important in the context of drug resistance, allowing growth and viability of cancer cells. Indeed, combined inhibition of PI3KAkt and MAPK pathways has shown efficacy in preclinical models, ${ }^{43-46}$ and could be of interest in the management of imatinib-resistant GISTs.

Considering the lack of acquired PI3KCA mutations, we further evaluated the hypothesis that BYL719 resistance could be mediated by epigenetic mechanisms. Studies reported that epigenetic modifications may represent alternative mechanisms to evade the pharmacological response. ${ }^{47-49}$ In this context, different trials are evaluating epigenetic therapies as drugresistance modulators in solid tumors. ${ }^{47}$ With regard to GISTs, in recent years epigenetic treatments raised among the future perspective $e^{50,51}$ alternatively to TKIs and/or to bypass TKI resistance. However, the results are still at an early stage, and further investigations are essential in this novel field. Consequently, we performed deep epigenomic characterization of these two in vitro models, looking for novel mechanisms of acquired resistance and potential druggable targets in GISTs. For this purpose, we performed miRNA profiling, identifying 13 miRNAs significantly deregulated in resistant cells. Among the pathways potentially modulated by these miRNAs, the most significant and those with the greatest number of target genes were PI3K/ AKT-MAPK, and RAS cascades, which are involved in BYL719's mechanism of action. Subsequently, integration of miRNA arrays and GEP revealed five miRNAs targeting eight genes (TRIB1, GAB2, SNX10, $S A M D 12, J U N B, G P C 3$, and SOX4). Among those genes, TRIB1 is a downstream effector of PI3K, and $G A B 2^{52}$ cooperates with the PI3K-AKT pathway in promoting malignant behavior in cells. ${ }^{53,54}$ 
Subsequently, we integrated methylation and GEP, identifying two networks of interest involving TSHZ2 and PSAT1. The TSHZ2 gene resulted in downregulated BYL719-resistant cells compared to sensitive ones. TSHZ2, a zinc-finger homeobox nuclear protein, is supposed to be a tumor suppressor and is downregulated in breast cancer. ${ }^{55}$ We speculated that TSHZ2 might bind transcriptional regulators that control the expression of crucial genes in tumorigenesis and resistance acquisition. Moreover, PSAT1 was upregulated in BYL719-resistant cells, and concomitant hypomethylation on the PSAT1 promoter was found. However, on integration of miRNA and methylation profiles with gene-expression analysis, we pinpointed overexpression of PSAT1 associated with both promoter hypomethylation and miR125b-5p downregulation. In particular, PSAT1 overexpression in BYL719-resistant cell lines could represent an advantage for resistant cells, which have to find novel ploys to live.

Here, we showed that both methylation and miRNA modulated PSAT1 overexpression. Additional studies are needed to clarify if the epigenetic mechanisms act concurrently or in a mutual manner, with the final goal of increasing PSAT1 levels, which confer a metabolic-related growth advantage to tumor cells. PSAT1 overexpression is involved in drug resistance in different tumors, including colorectal cancer, melanoma, pancreatic cancer, and non-small-cell lung cancer. ${ }^{56,57}$ PSAT1 belongs to the serine biosynthesis pathway, which has a key role in nucleotide and amino-acid metabolism. Levels of enzymes involved in serine synthesis have been shown to increase under conditions of DNA damage and genomic instability. ${ }^{57,58}$ In addition, a recent study reported that serine supports one-carbon metabolism and proliferation of cancer cells. ${ }^{59}$ Moreover, different evidence suggests that the serine pathway is crucial in cancer metabolism, using glycolysis-derived glucose for serine production and tumor growth. ${ }^{60-64}$ In addition to these studies, which clearly ascribed to PSAT1 a role in tumorigenesis, a recent study on esophageal squamous-cell carcinoma patients linked PSAT1 overexpression to upregulation of the PI3K-AKT-GSK3 $\beta$-Snail pathway. ${ }^{65}$ Therefore, targeting PSAT1 might have potential therapeutic implication in GIST patients.

In conclusion, we identified novel epigenomic mechanisms of pharmacological resistance in GISTs, suggesting the existence of pathways involved in drug resistance and alternative to acquired mutations. We are aware that prolonged exposure and high BYL719 concentrations used to generate the resistant models can be de facto a substantial source of numerous off-target effects. However, prolonged inhibition of the PI3K pathway is probably accompanied by profound transcriptomic, proteomic, and metabolomic changes involved in drug resistance. Therefore, epigenomics should be taken into account as an alternative adaptive mechanism. Currently we do not have access to GIST patients in treatment with BYL719, and thus we are not able to verify the hypothesis in vivo. However, we consider these results intriguing, particularly the involvement of H19 and PSTA1 in GIST resistance, which might represent novel druggable targets for GIST patients.

\section{Acknowledgments}

This work was supported by the Ministry of Education, University, and Research of Italy (MIUR; grant 2015Y3C5KP_002 to SA). Gloria Ravegnini is supported by an MSD Italia fellowship granted by and on behalf of MSD and L'Oréal-UNESCO for Women in Science. Giulia Sammarini is supported by Fondazione Famiglia Parmiani.

\section{Disclosure}

The authors report no conflicts of interest in this work.

\section{References}

1. Mei L, Smith SC, Faber AC, et al. Gastrointestinal stromal tumors: the GIST of precision medicine. Trends Cancer. 2018;4(1):74-91. doi:10.1016/j.trecan.2017.11.006

2. Corless CL, Barnett CM, Heinrich MC. Gastrointestinal stromal tumours: origin and molecular oncology. nat rev cancer. 2011;11 (12):865-878. doi:10.1038/nrc3143

3. Serrano C, George S. Recent advances in the treatment of gastrointestinal stromal tumors. Ther Adv Med Oncol. 2014;6(3):115-127. doi: $10.1177 / 1758834014522491$

4. Demetri GD, van Oosterom AT, Garrett CR, et al. Efficacy and safety of sunitinib in patients with advanced gastrointestinal stromal tumour after failure of imatinib: a randomised controlled trial. Lancet (London, England). 2006;368(9544):1329-1338. doi:10.1016/S01406736(06)69446-4

5. Demetri GD, Reichardt P, Kang Y-K, et al. Efficacy and Safety of regorafenib for advanced gastrointestinal stromal tumours after failure of imatinib and sunitinib (GRID): an international, multicentre, randomised, placebo-controlled, phase 3 trial. Lancet. 2013;381 (9863):295-302. doi:10.1016/S0140-6736(12)61857-1

6. Hemming ML, Heinrich MC, Bauer S, George S. Translational insights into gastrointestinal stromal tumor and current clinical advances. Ann Oncol Off J Eur Soc Med Oncol. 2018. doi:10.1093/ annonc/mdy309

7. Liegl B, Kepten I, Le C, et al. Heterogeneity of Kinase inhibitor resistance mechanisms in GIST. $J$ Pathol. 2008;216(1):64-74. doi:10.1002/path.2382

8. Yoo C, Ryu M-H, Jo J, Park I, Ryoo B-Y, Kang Y-K. Efficacy of imatinib in patients with platelet-derived growth factor receptor alphamutated gastrointestinal stromal tumors. Cancer Res Treat. 2016;48 (2):546-552. doi:10.4143/crt.2015.015 
9. Cassier PA, Fumagalli E, Rutkowski P, et al. Outcome of patients with platelet-derived growth factor receptor alpha-mutated gastrointestinal stromal tumors in the tyrosine kinase inhibitor era. Clin Cancer Res. 2012;18(16):4458-4464. doi:10.1158/1078-0432.CCR11-3025

10. Van Looy T, Wozniak A, Floris G, et al. Phosphoinositide 3-kinase inhibitors combined with imatinib in patient-derived xenograft models of gastrointestinal stromal tumors: rationale and efficacy. Clin Cancer Res. 2014;20(23):6071-6082. doi:10.1158/1078-0432.CCR14-1823

11. Fritsch C, Huang A, Chatenay-Rivauday C, et al. Characterization of the novel and specific PI3K $\alpha$ inhibitor NVP-BYL719 and development of the patient stratification strategy for clinical trials. Mol Cancer Ther. 2014;13(5):1117-1129. doi:10.1158/1535-7163.MCT-13-0865

12. Serrano C, Wang Y, Mariño-Enríquez A, et al. KRAS and KIT gatekeeper mutations confer polyclonal primary imatinib resistance in GI stromal tumors: relevance of concomitant phosphatidylinositol 3-kinase/AKT dysregulation. $J$ Clin Oncol. 2015;33(22):e93-6. doi:10.1200/JCO.2013.48.7488

13. Bauer S, Duensing A, Demetri GD, Fletcher JA. KIT oncogenic signaling mechanisms in imatinib-resistant gastrointestinal stromal tumor: PI3-kinase/AKT is a crucial survival pathway. Oncogene. 2007;26(54):7560-7568. doi:10.1038/sj.onc.1210558

14. Vivanco I, Sawyers CL. The phosphatidylinositol 3-kinase AKT pathway in human cancer. Nat Rev Cancer. 2002;2(7):489-501. doi: $10.1038 / \mathrm{nrc} 839$

15. Patel S. Exploring novel therapeutic targets in GIST: focus on the PI3K/Akt/MTOR pathway. Curr Oncol Rep. 2013;15(4):386-395. doi:10.1007/s11912-013-0316-6

16. Ríos-Moreno MJ, Jaramillo S, Díaz-Delgado M, et al. Differential activation of MAPK and PI3K/AKT/MTOR pathways and IGF1R expression in gastrointestinal stromal tumors. Anticancer Res. 2011;31(9):3019-3025.

17. Ulukaya E, Colakogullari M, Wood EJ. Interference by anti-cancer chemotherapeutic agents in the MTT-tumor chemosensitivity assay. Chemotherapy. 2004;50(1):43-50. doi:10.1159/000077285

18. Kumar P, Henikoff S, Ng PC. predicting the effects of coding non-synonymous variants on protein function using the SIFT algorithm. Nat Protoc. 2009;4(7):1073-1081. doi:10.1038/nprot.2009.86

19. Adzhubei IA, Schmidt S, Peshkin L, et al. A method and server for predicting damaging missense mutations. Nat Methods. 2010;7 (4):248-249. doi:10.1038/nmeth0410-248

20. Schwarz JM, Cooper DN, Schuelke M, Seelow D. MutationTaster2: mutation prediction for the deep-sequencing age. Nat Methods 2014;11(4):361-362. doi:10.1038/nmeth.2890

21. Barault L, Amatu A, Siravegna G, et al. Discovery of methylated circulating DNA biomarkers for comprehensive non-invasive monitoring of treatment response in metastatic colorectal cancer. Gut. 2017. doi:10.1136/gutjnl-2016-313372 gutjnl-2016-313372.

22. Moran S, Arribas C, Esteller M. Validation of a DNA methylation microarray for $850,000 \mathrm{cpg}$ sites of the human genome enriched in enhancer sequences. Epigenomics. 2016;8(3):389-399. doi:10.2217/ epi.15.114

23. Omarini C, Filieri ME, Bettelli S, et al. Mutational profile of metastatic breast cancer tissue in patients treated with exemestane plus everolimus. Biomed Res Int. 2018;2018:3756981. doi:10.1155/2018/3756981

24. Tessitore A, Bruera G, Mastroiaco V, et al. KRAS and 2 rare PI3KCA mutations coexisting in a metastatic colorectal cancer patient with aggressive and resistant disease. Hum Pathol. 2018;74:178-182. doi:10.1016/j.humpath.2018.01.021

25. Lai K, Killingsworth MC, Lee CS. Gene of the Month: PIK3CA. J Clin Pathol. 2015;68(4):253-257. doi:10.1136/jclinpath-2015-202885

26. Samuels Y, Wang Z, Bardelli A, et al. High frequency of mutations of the PIK3CA gene in human cancers. Science. 2004;304(5670):554 doi: $10.1126 /$ science. 1096502
27. Vlachos IS, Zagganas K, Paraskevopoulou MD, et al. DIANAMiRPath v3.0: deciphering MicroRNA function with experimental support. Nucleic Acids Res. 2015;43(W1):W460-W466. doi:10.1093/ nar/gkv403

28. Chou C-H, Shrestha S, Yang C-D, et al. MiRTarBase update 2018: a resource for experimentally validated MicroRNA-target interactions. Nucleic Acids Res. 2018;46(D1):D296-D302. doi:10.1093/nar/gkx1067

29. Mühlenberg T, Zhang Y, Wagner AJ, et al. Inhibitors of deacetylases suppress oncogenic KIT signaling, acetylate HSP90, and induce apoptosis in gastrointestinal stromal tumors. Cancer Res. 2009;69 (17):6941-6950. doi:10.1158/0008-5472.CAN-08-4004

30. Heinrich MC, Maki RG, Corless CL, et al. Primary and secondary kinase genotypes correlate with the biological and clinical activity of sunitinib in imatinib-resistant gastrointestinal stromal tumor. J Clin Oncol. 2008;26(33):5352-5359. doi:10.1200/JCO.2007.15.7461

31. Wardelmann E, Merkelbach-Bruse S, Pauls K, et al. Polyclonal evolution of multiple secondary KIT mutations in gastrointestinal stromal tumors under treatment with imatinib mesylate. Clin Cancer Res. 2006;12(6):1743-1749. doi:10.1158/1078-0432.CCR-05-1211

32. Beretta GL, Cassinelli G, Pennati M, Zuco V, Gatti L. Overcoming $\mathrm{ABC}$ transporter-mediated multidrug resistance: the dual role of tyrosine kinase inhibitors as multitargeting agents. Eur $J$ Med Chem. 2017;142:271-289. doi:10.1016/j.ejmech.2017.07.062

33. Choi YH, Yu A-M. ABC transporters in multidrug resistance and pharmacokinetics, and strategies for drug development. Curr Pharm Des. 2014;20(5):793-807. doi:10.2174/138161282005140214165212

34. Klukovits A, Krajcsi P. Mechanisms and therapeutic potential of inhibiting drug efflux transporters. Expert Opin Drug Metab Toxicol. 2015;11(6):907-920. doi:10.1517/17425255.2015.1028917

35. Raveh E, Matouk IJ, Gilon M, Hochberg A. The H19 long non-coding RNA in cancer initiation, progression and metastasis a proposed unifying theory. Mol Cancer. 2015;14(1):184. doi:10.1186/s12943-015-0458-2

36. Huarte M. The emerging role of LncRNAs in cancer. Nat Med. 2015;21(11):1253-1261. doi:10.1038/nm.3981

37. Yang Q, Wang X, Tang C, Chen X, He J. H19 promotes the migration and invasion of colon cancer by sponging MiR-138 to upregulate the expression of HMGA1. Int J Oncol. 2017;50(5):1801-1809. doi:10.3892/ijo.2017.3941

38. Li H, Yu B, Li J, et al. Overexpression of LncRNA H19 enhances carcinogenesis and metastasis of gastric cancer. Oncotarget. 2014;5 (8):2318-2329. doi:10.18632/oncotarget.1913

39. Xue L, Wang Y, Yue S, Zhang J. The expression of miRNA-221 and miRNA-222 in gliomas patients and their prognosis. Neurol Sci. 2017;38(1):67-73. doi:10.1007/s10072-016-2710-y

40. Chi P, Chen Y, Zhang L, et al. ETV1 is a lineage survival factor that cooperates with KIT in gastrointestinal stromal tumours. Nature. 2010;467(7317):849-853. doi:10.1038/nature09409

41. Yang W, Redpath R, Zhang C, Ning N. Long non-coding RNA H19 promotes the migration and invasion of colon cancer cells via MAPK signaling pathway. Oncol Lett. 2018;16(3):3365-3372. doi:10.3892/ ol.2018.9052

42. Tolcher AW, Peng W, Calvo E. Rational approaches for combination therapy strategies targeting the MAP kinase pathway in solid tumors. Mol Cancer Ther. 2018;17(1):3-16. doi:10.1158/1535-7163.MCT-170349

43. Aksamitiene E, Kiyatkin A, Kholodenko B.N. Cross-talk between mitogenic Ras/MAPK and survival PI3K/Akt pathways: a fine balance. Biochem Soc Trans. 2012;40(5):139-146. doi:10.1042/ BST20110609

44. Alonso N, Diaz Nebreda A, Monczor F, et al. PI3K pathway is involved in ERK signaling cascade activation by histamine H2R agonist in HEK293T cells. Biochim Biophys Acta. 2016;1860 (9):1998-2007. doi:10.1016/j.bbagen.2016.06.016 
45. Serra V, Scaltriti M, Prudkin L, et al. PI3K Inhibition Results in Enhanced HER Signaling and Acquired ERK Dependency in HER2-Overexpressing Breast Cancer. Oncogene. 2011;30 (22):2547-2557. doi:10.1038/onc.2010.626

46. Engelman JA, Chen L, Tan X, et al. Effective use of PI3K and MEK inhibitors to treat mutant kras G12D and PIK3CA H1047R murine lung cancers. Nat Med. 2008;14(12):1351-1356. doi:10.1038/nm.1890

47. Brown R, Curry E, Magnani L, Wilhelm-Benartzi CS, Borley J. Poised epigenetic states and acquired drug resistance in cancer. Nat Rev Cancer. 2014;14(11):747-753. doi:10.1038/nrc3819

48. Knoechel B, Roderick JE, Williamson KE, et al. An epigenetic mechanism of resistance to targeted therapy in $\mathrm{T}$ cell acute lymphoblastic leukemia. Nat Genet. 2014;46(4):364-370. doi:10.1038/ng.2913

49. Salgia R, Kulkarni P. The genetic/non-genetic duality of drug 'resistance' in cancer. Trends Cancer. 2018;4(2):110-118. doi:10.1016/j. trecan.2018.01.001

50. Nervi C, De Marinis E, Codacci-Pisanelli G. Epigenetic treatment of solid tumours: a review of clinical trials. Clin Epigenetics. 2015;7:127. doi:10.1186/s13148-015-0157-2

51. Bauer S, Hilger RA, Mühlenberg T, et al. Phase I study of panobinostat and imatinib in patients with treatment-refractory metastatic gastrointestinal stromal tumors. $B r \quad J \quad$ Cancer. 2014;110 (5):1155-1162. doi:10.1038/bjc.2013.826

52. De Marco C, Laudanna C, Rinaldo N, et al. Specific Gene expression signatures induced by the multiple oncogenic alterations that occur within the PTEN/PI3K/AKT pathway in lung cancer. PLoS One. 2017;12(6):e0178865. doi:10.1371/journal.pone.0178865

53. Wang Y, Sheng Q, Spillman MA, Behbakht K, Gu H. Gab2 regulates the migratory behaviors and e-cadherin expression via activation of the pi3k pathway in ovarian cancer cells. Oncogene. 2012;31 (20):2512-2520. doi:10.1038/onc.2011.435

54. Wang WJ, Mou K, Wu XF, et al. Grb2-associated binder 2 silencing impairs growth and migration of $\mathrm{H} 1975$ cells via modulation of PI3K-Akt signaling. Int $J$ Clin Exp Pathol. 2015;8 (9):10575-10584.

55. Yamamoto M, Cid E, Bru S, Yamamoto F. Rare and frequent promoter methylation, respectively, of TSHZ2 and 3 genes that are both downregulated in expression in breast and prostate cancers. PLoS One. 2011;6(3):e17149. doi:10.1371/journal.pone.0017149
56. Vié N, Copois V, Bascoul-Mollevi C, et al. Overexpression of phosphoserine aminotransferase PSAT1 stimulates cell growth and increases chemoresistance of colon cancer cells. Mol Cancer. 2008;7(1):14. doi:10.1186/1476-4598-7-14

57. Ross KC, Andrews AJ, Marion CD, Yen TJ, Bhattacharjee V. Identification of the serine biosynthesis pathway as a critical component of BRAF inhibitor resistance of melanoma, pancreatic, and nonsmall cell lung cancer cells. Mol Cancer Ther. 2017;16 (8):1596-1609. doi:10.1158/1535-7163.MCT-16-0798

58. Markkanen E, Fischer R, Ledentcova M, Kessler BM, Dianov GL. Cells deficient in base-excision repair reveal cancer hallmarks originating from adjustments to genetic instability. Nucleic Acids Res. 2015;43(7):3667-3679. doi:10.1093/nar/gkv222

59. Labuschagne CF, van Den Broek NJF, Mackay GM, Vousden KH, Maddocks ODK. Serine, but not glycine, supports one-carbon metabolism and proliferation of cancer cells. Cell Rep. 2014;7 (4):1248-1258. doi:10.1016/j.celrep.2014.04.045

60. Schulze A, Harris AL. How cancer metabolism is tuned for proliferation and vulnerable to disruption. Nature. 2012;491 (7424):364-373. doi:10.1038/nature 11706

61. Luo J. Cancer's sweet tooth for serine. Breast Cancer Res. 2011;13 (6):317. doi: $10.1186 / \mathrm{bcr} 2932$

62. De Marchi T, Timmermans MA, Sieuwerts AM, et al. Phosphoserine aminotransferase 1 is associated to poor outcome on tamoxifen therapy in recurrent breast cancer. Sci Rep. 2017;7(1):2099. doi:10.1038/ s41598-017-02296-w

63. Locasale JW, Grassian AR, Melman T, et al. Phosphoglycerate dehydrogenase diverts glycolytic flux and contributes to oncogenesis. Nat Genet. 2011;43(9):869-874. doi:10.1038/ng.890

64. Possemato R, Marks KM, Shaul YD, et al. Functional genomics reveal that the serine synthesis pathway is essential in breast cancer. Nature. 2011;476(7360):346-350. doi:10.1038/nature10350

65. Liu B, Jia Y, Cao Y, et al. Overexpression of phosphoserine aminotransferase 1 (PSAT1) predicts poor prognosis and associates with tumor progression in human esophageal squamous cell carcinoma. Cell Physiol Biochem. 2016;39(1):395-406. doi: $10.1159 / 000445633$ 


\section{Supplementary materials}

Table SI List of genes differentially expressed in parental and resistant cell lines

\begin{tabular}{|c|c|c|c|c|c|c|c|}
\hline \multicolumn{4}{|c|}{ Upregulated genes in BYL7 I9-resistant cell lines } & \multicolumn{4}{|c|}{ Downregulated genes in BYL7 I9-resistant cell lines } \\
\hline & LogFC & P-value & FDR & & LogFC & P-value & FDR \\
\hline$H 19$ & 2.23 & $8.61^{-12}$ & $1.08^{-7}$ & Cl6ORF89 & -2.21 & 0 & 0.000001 \\
\hline RAB3IP & 3.23 & $1.19^{-9}$ & $3.93^{-6}$ & MXRA5 & -1.90 & 0 & 0.000004 \\
\hline ANXA3 & 1.81 & $8.24^{-8}$ & 0.000148 & BRINPI & -3.82 & 0 & 0.000121 \\
\hline$L X N$ & 2.57 & $2.9^{-7}$ & 0.000304 & CUX2 & -2.27 & 0 & 0.000215 \\
\hline $\mathrm{CHACl}$ & 2.41 & $1^{-6}$ & $0.00084 I$ & SLC4A7 & -1.57 & 0 & 0.000215 \\
\hline TNFSFIO & 3.12 & $1.53^{-6}$ & 0.001043 & PCSK6 & -1.78 & 0 & 0.000215 \\
\hline CLMN & 2.40 & $1.8^{-6}$ & 0.001078 & SGKI & -2.46 & 0.000001 & 0.000535 \\
\hline ACSM3 & 2.26 & $5.71^{-6}$ & 0.002987 & GPC2 & -1.79 & 0.000001 & 0.001010 \\
\hline PDE5A & 1.52 & $9.67^{-6}$ & 0.004328 & FNI & -1.54 & 0.000003 & 0.001451 \\
\hline DSG2 & 2.60 & $1.39^{-5}$ & 0.005618 & RHBDFI & -1.43 & 0.000007 & 0.003245 \\
\hline ASNS & 1.30 & $1.58^{-5}$ & 0.00619 & SAMDII & -1.38 & 0.000008 & 0.003622 \\
\hline PSATI & 1.48 & $1.96^{-5}$ & 0.007033 & VCAMI & -1.67 & 0.000017 & 0.006486 \\
\hline SNXIO & 2.00 & $2.5 I^{-5}$ & 0.008495 & ID3 & -1.69 & 0.000019 & 0.006898 \\
\hline LMO4 & 1.29 & $2.64^{-5}$ & 0.008495 & SMAD9 & -1.28 & 0.000029 & 0.008963 \\
\hline SEMA3C & 2.39 & $3^{-5}$ & 0.009034 & $S L C I 4 A I$ & -3.15 & 0.000030 & 0.009034 \\
\hline GLRB & 2.02 & $3.22^{-5}$ & 0.009306 & $T S H Z 2$ & -2.18 & 0.000033 & 0.009306 \\
\hline$A D D 2$ & 1.62 & $4.99^{-5}$ & 0.012541 & MME & -1.47 & 0.000035 & 0.009897 \\
\hline EGRI & 2.46 & $7.05^{-5}$ & 0.017026 & RPII-5407.I & -2.01 & 0.000041 & 0.010885 \\
\hline MAL2 & 2.82 & $7.84^{-5}$ & 0.017893 & PHF2P2 & -1.39 & 0.000042 & 0.011066 \\
\hline PRIMAI & 2.06 & $8.88^{-5}$ & 0.018896 & TYRO3 & -1.42 & 0.000097 & 0.019908 \\
\hline$T B C I D 2 B$ & 1.47 & $9.25^{-5}$ & 0.019348 & NUAKI & -1.45 & 0.000105 & 0.020765 \\
\hline GAB2 & 2.19 & 0.000108 & 0.020816 & PHLDB2 & -1.64 & 0.000106 & 0.020765 \\
\hline PROCR & 1.89 & 0.000129 & 0.023094 & DACT3 & -1.43 & 0.000104 & 0.020765 \\
\hline$|C A|$ & 2.05 & 0.000132 & 0.023302 & GTF2IP3 & -2.01 & 0.000109 & 0.020816 \\
\hline PRKCQ-ASI & 1.56 & 0.000142 & 0.024358 & ID2 & -1.25 & 0.000117 & 0.021688 \\
\hline DOCK9 & 1.77 & 0.000171 & 0.0276 & EFNB2 & -1.59 & 0.000137 & $0.0238 I I$ \\
\hline LAMP3 & 1.22 & 0.000232 & 0.035097 & IQGAP2 & -1.14 & 0.000144 & 0.024358 \\
\hline TRIBI & 1.25 & 0.000232 & 0.035097 & $R B M / 8$ & -1.17 & 0.000170 & 0.027600 \\
\hline TBCID8B & 1.62 & 0.000266 & 0.038374 & SOSTDCI & -1.84 & 0.000189 & 0.030100 \\
\hline SFRPI & 1.10 & 0.000358 & 0.048283 & $F 2 R$ & -1.48 & 0.000225 & 0.034926 \\
\hline TNFAIP8 & 1.29 & 0.000407 & 0.052689 & $A D G R D I$ & -1.54 & 0.000262 & 0.038374 \\
\hline SAMDI 2 & 2.06 & 0.000424 & 0.053714 & GAREM & -1.46 & 0.000297 & 0.042399 \\
\hline JUNB & 1.43 & 0.000444 & 0.055782 & sox 4 & -1.23 & 0.000306 & 0.043207 \\
\hline DAAM2 & 1.21 & 0.000483 & 0.05878 & SMAD6 & -1.21 & 0.000350 & 0.047754 \\
\hline IGSF3 & 1.13 & 0.000487 & 0.05878 & SH3BP5-ASI & -1.66 & 0.000347 & 0.047754 \\
\hline$L G I 2$ & 1.90 & 0.000521 & 0.061597 & PREX2 & -1.06 & 0.000369 & 0.049242 \\
\hline CALB2 & 1.72 & 0.000525 & 0.061597 & $B C R P 3$ & -1.76 & 0.000420 & 0.053714 \\
\hline PDE3B & 1.39 & 0.000552 & 0.063588 & COLI6AI & -1.15 & 0.000449 & 0.055782 \\
\hline GPC3 & I.7I & 0.000574 & 0.064953 & PDLIM3 & -1.45 & 0.000463 & 0.056947 \\
\hline TNFRSFI 9 & 2.17 & 0.000571 & 0.064953 & ADAMTS2 & -1.65 & 0.000512 & 0.061195 \\
\hline FOXP2 & 1.57 & 0.00061 & 0.06843 & WTIP & -1.16 & 0.000531 & 0.061674 \\
\hline BST2 & 1.18 & 0.000619 & 0.068795 & MYOIB & -1.27 & 0.000626 & 0.068960 \\
\hline HUNK & 1.30 & 0.000647 & 0.070631 & SORBSI & -1.55 & 0.000703 & 0.075396 \\
\hline FAM $19 A 4$ & 1.76 & 0.000709 & 0.075396 & PPARGCIB & -1.01 & 0.000794 & 0.081682 \\
\hline$A R R B I$ & 1.27 & 0.000737 & 0.07712 & ATOH8 & -2.31 & 0.000808 & 0.082507 \\
\hline$C C D C / 8 I$ & 1.55 & 0.000893 & $0.08825 I$ & ZDHHCII & -1.33 & 0.000832 & 0.084194 \\
\hline GLTSCR2 & 0.98 & 0.001004 & 0.098527 & CTGF & -1.16 & 0.000869 & 0.087235 \\
\hline FOS & 2.77 & 0.001014 & 0.098663 & & & & \\
\hline
\end{tabular}

Abbreviation: FDR, fold discovery rate. 

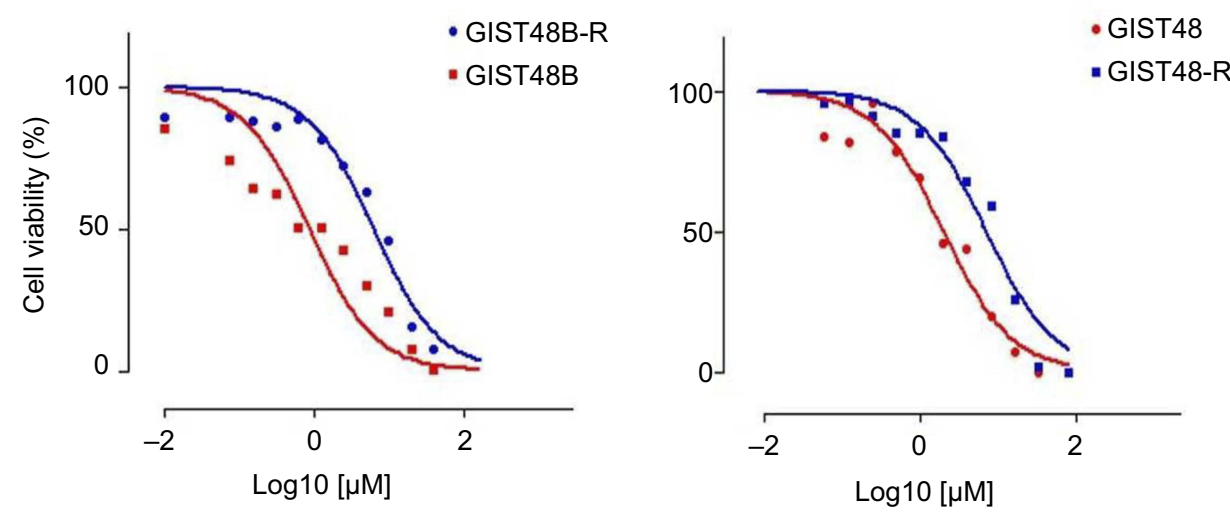

Figure SI MTT assay.

Notes: Cell viability in GIST48 and GIST48B (red curves) and BYL719-resistant counterparts (blue curves).

\section{Publish your work in this journal}

Cancer Management and Research is an international, peer-reviewed open access journal focusing on cancer research and the optimal use of preventative and integrated treatment interventions to achieve improved outcomes, enhanced survival and quality of life for the cancer patient.
The manuscript management system is completely online and includes a very quick and fair peer-review system, which is all easy to use. Visit http://www.dovepress.com/testimonials.php to read real quotes from published authors. 